\title{
Multi-parameter optimization of lightweight composite triangular truss structure based on response surface methodology
}

Su Jua,*, R. A. Shenoi ${ }^{b}$, Dazhi Jianga, A. J. Sobey

${ }^{a}$ College of Aerospace and Materials Engineering, National University of Defense Technology, Changsha 410073, China

b School of Engineering Science, University of Southampton, Southampton SO17 1BJ, $U K$

\section{Abstract}

A multi-parameter optimization approach for a lightweight FRP composite triangular truss under nonlinear structural response constraints is presented in this paper. The composite truss exhibited bilinear behavior under three-point bending which was observed by both experimental and numerical investigation. Sensitivity analysis was conducted to evaluate the effects of geometric parameters on the flexural performance of the composite truss. A response surface, developed from a finite element analysis, was employed to replicate the outputs of nonlinear structural responses with respect to four key geometric parameters. Gradientbased and Genetic Algorithm optimization processes were selected and

* Corresponding author at: College of Aerospace and Materials Engineering, National University of Defense Technology, Changsha 410073, P.R. China. Tel.: +86 (0)731 84576315; fax: +86 (0)73184576578.

E-mail address: jusu.nudt@gmail.com (Su Ju). 
implemented from the MATLAB Optimization Toolbox. Final adjustments to the optimal design, obtained using MATLAB, were made according to the results of sensitivity analysis. The results show that significant improvement in terms of weight saving were achieved by adopting the proposed optimization design procedure.

\section{Keywords}

A. Polymer-matrix composites (PMCs)

B. Mechanical properties

C. Finite element analysis (FEA)

D. Statistical method

\section{Introduction}

Fiber reinforced polymer (FRP) composite materials offer potential savings in structural weight and their structural applications have increased due to their excellent specific stiffness, specific strength, sufficient durability, and reduced energy consumption [1]. Trusses, as an efficient structural form, have been widely used in civil engineering and industry while composite truss structures have been widely applied in advanced transportation technologies. As an example, Schtüze [2] has used the triangular cross section lightweight carbon fiber composite truss in the Zeppelin airship.

Recent developments in manufacturing technologies have allowed composite truss structures to be integrally fabricated using filament winding with the same 
structural element repetitively appearing along the length. These new types of continuous filament FRP composite truss structures, also known as beam-like lattice structures, have attracted considerable attention due to their high efficiency in load distribution, long span, good integrity (few joints), and high specific stiffness and strength. An IsoTruss ${ }^{\mathrm{TM}}$ structure has been developed by Jensen et al. [3-5] whose manufacturing technique and mechanical properties have been researched over many years. Load transfer paths are mainly along the members of these truss structures. Continuous unidirectional FRP composite materials are particularly well suited for manufacturing these composite truss structures as the high directionality of unidirectional FRP composite materials allows the majority of material's stiffness and strength to be located and directed along the load transfer path. The high specific stiffness and strength of unidirectional FRP composite materials are fully utilized in these trusses, thus weight saving is achieved.

Further weight savings of the FRP composite truss structures can be achieved by geometric optimization. Optimization design for composite structures is a difficult task because of the numerous design variables and state variables which have to be simultaneously taken into account. Optimization procedures of composite structure are often developed for specific applications for which the geometry of the structure is simple. This is mainly due to the fact that the explicit analytic expressions of structural response are available in these cases [6]. Structural responses, such as stress, strain, load and displacement, are usually employed as constraints in an optimization process. Industrial composite structures exhibit more complex geometries and loading cases in which designers have to use finite 
element analysis (FEA) to obtain the structural response. For these structures, the approach of coupling optimization and finite element (FE) calculation is naturally adopted. Paluch et al. [6] and Walker et al. [7] have combined a finite element program and a genetic algorithm to optimize the ply thickness and orientation of composite laminated structure. Liu and Lu [8] have developed a multi-parameter optimization procedure for ultra-lightweight truss core sandwich panels by combining standard finite element analysis with structural system profile analysis and multi-factor optimization techniques.

The method of combining finite element analysis (commercial FE code) with standard optimization process work well for cases where the structural responses are under static linear mechanical behavior allowing easy extraction of data automatically, using an optimization program. For structures with nonlinear mechanical behavior, the solution and extraction of structural responses becomes much more time consuming and difficult when using FEA. In addition, for problems associated with FE nonlinear solutions, convergence difficulties due to large deformations causing mesh distortion were encountered which led to failure or low efficiency of the optimization process. Consequently, new means were explored and developed to obtain the values of structural response for the optimization process.

Researchers in the mid 1970s began to explore the use of approximation concepts as a device to reduce the number of structural analyses, and hence the total computational effort. Response surface methodology (RSM), as a robust global approximation method, is more capable of satisfactorily predicting structural 
response over a wide range of design space [9]. RSM is a set of techniques that encompasses:

(1) Designing a set of experiments that will yield adequate and reliable measurements of the response of interest;

(2) Determining a mathematical model that best fits the data collected from the design chosen in (1), by conducting appropriate tests of hypotheses concerning the model's parameters; and

(3) Determining the optimal settings of the experimental factors that produce the maximum (or minimum) value of the response.

RSM was formally developed by G.E.P Box and K.B. Wilson et al. [10] from 1951 and initially used to explore the relationship between the yield of chemical process and a set of input variables presumed to influence the yield. Since the pioneering work of Box and his colleagues, RSM has been successfully applied in chemical engineering, industrial development, biological research, and computer simulation [11]. Abu-Odeh et al. [9] has constructed the response surfaces of vertical displacement and three bending moment components at a point on a composite plate, which have been used in the optimization of a composite laminated plate, instead of an exact FE analysis, during constraint evaluation. Chen et al. [12] have combined the response surface method and a first order reliability method to calculate the safety index and failure probability. A discussion on the sensitivity of each variable that effects the reliability has also been included. A new method of experimental design has been proposed by Todoroki and Ishikawa [13] to obtain a response surface of buckling load of laminated composites. Lanzi and Giavotto [14] 
have developed a multi-objective optimization procedure for the design of composite stiffened panels and the response surfaces have been used to approximate the post-buckling behavior of the composite stiffened panels.

Previous optimization work employing response surface methods mainly focused on the composite laminated plates or stiffened panels. Limited studies have been performed on the long span composite truss structures under nonlinear structural response constraints. This paper proposes a multi-parameter optimization design approach combining the standard optimization program and a response surface methodology to minimize the structural weight of lightweight FRP composite truss structure under nonlinear structural response constraints.

\section{Design optimization framework}

This study has developed a multi-parameter optimization procedure of combining RSM and a standard program to optimize complex composite structures under nonlinear structural response constraints. The long span FRP composite truss with triangular cross section is taken as an instance to illustrate the proposed optimization procedure.

The flow chart in Fig. 1 depicts the proposed design optimization framework for the complex composite structures with nonlinear structural response. To ensure the optimized result is practical, it is necessary to validate the numerical results by experimental studies before the FE model is formally employed. Further modification of the FE model is required until good agreement is achieved between the results of the experiment and FEA. A parametric modeling technique is adopted in building the FE model as it will facilitate the sensitivity analysis and 
sampling steps that follow. Sensitivity analysis is performed to investigate effects of design variables on structural responses which will also provide a design guideline for choosing actual design variable values. RSM is used to design 'numerical experiments' to collect sample points data as parsimoniously as possible corresponding to the whole design space. This while provides sufficient information to construct a response surface model as accurately as possible. The computation and extraction of the sample point data is the most time consuming work during the whole optimization process. However, for complex composite structures, significant time saving is still achieved compared with embedding the FEA into the optimization program, especially for nonlinear solutions. These sample points are then adopted to construct a response surface model. Errors between the structural responses predicted by the response surface model and FEA are then assessed. The accuracy of the response surface model can be improved by increasing sample points at specific regions of interest. When the response surface model is working with satisfactory accuracy it is then connected to some standard optimization programs (e.g. MATLAB Optimization Toolbox ${ }^{\mathrm{TM}}$ ). Compared to the FE solution, RSM is an approximation method and some errors are inevitable. RSM based optimization often leads to results that are close to the true global optimum but may never reach this exact value. The optimized results may need to be adjusted, by referring to a sensitivity analysis, before a final optimal design is obtained.

For the case of a triangular cross section lightweight FRP composite truss structure, four geometric parameters, circumscribing diameter of triangular cross section $D$, longitudinal member diameter $d_{1}$, helical member diameter $d_{2}$, and bay number $N$, 
are simultaneously taken into account as design variables (see Fig. 2). Total length $L$ is fixed. The same structural element 'IXl' repeats along the length direction on three sides in this composite truss structure. It is of practical interest that the structural weight $W$ of the composite truss is considered as the objective to be minimized. In many applications, the critical condition for the long span composite truss structure is buckling under bending load. Thus the composite truss structure under three-point bending is considered as the critical loading condition. For many composite structural components it is desirable for them to withstand external loads without suffering excessive deformation. From this point of view, the ultimate load $L_{t}$ and the displacement under this load $S_{t}$ are taken as constraints. Consequently, the optimization design problem of minimizing structural weight of lightweight FRP composite truss structures subjected to nonlinear structural responses can be formulated as follows:

minimize structural weight:

$W=f\left(D, N, d_{1}, d_{2}\right)$

subject to ultimate load and displacement inequality constraints:

$L_{t}=g_{1}\left(D, N, d_{1}, d_{2}\right) \geq L_{t}{ }^{p}$,

$S_{t}=g_{2}\left(D, N, d_{1}, d_{2}\right) \leq S_{t}^{p}$

Geometry constraints are:

$D^{l} \leq D \leq D^{u}$,

$N^{l} \leq N \leq N^{u}(N=1,2,3, \ldots)$ 
$d_{1}{ }^{l} \leq d_{1} \leq d_{1}{ }^{u}$

$d_{2}{ }^{l} \leq d_{2} \leq d_{2}^{u}$,

where $f$ is objective function which can be written as:

$$
\begin{aligned}
W & =f\left(D, N, d_{1}, d_{2}\right) \\
& =3 \rho_{L} L \frac{\pi d_{1}^{2}}{4}+3(N+1) \rho_{H} \frac{\sqrt{3} D}{2} \frac{\pi d_{2}^{2}}{4}+6 N \rho_{H} \sqrt{\left(\frac{\sqrt{3} D}{2}\right)^{2}+\left(\frac{L}{N}\right)^{2}} \frac{\pi d_{2}^{2}}{4} .
\end{aligned}
$$

$\rho_{L}$ and $\rho_{H}$ are the density of the longitudinal and helical members of the composite truss, respectively. $g_{1}$ and $g_{2}$ are performance functions [15], describing the relationship between design variables and structural responses, which are always evaluated by analytical or numerical techniques [16] in optimization processes. For ultimate load and displacement constraints, $L_{t} p$ and $S_{t} t^{p}$ are prescribed a minimum and maximum allowable value respectively, chosen according to the practical application requirements. Symbols $l$ and $u$ in the geometry constraints denote the lower and upper bounds of the design variables which will be given based on the considerations of the feasibility of manufacturing process and the rationality of the composite structure.

Applying the proposed design optimization framework to this practical case, three main research steps are included.

1) A 6 m long, triangular cross section, GF/epoxy lightweight FRP composite truss specimen is made and tested under three-point bending. FEA corresponding to the experimental study is conducted simultaneously. Experimental and numerical results are compared. The critical structural responses, ultimate load $L_{t}$ and 
corresponding displacement $S_{t}$, are identified and extracted as constraints for the optimization loops. After confirming that a good agreement is achieved between the experimental and numerical results, a parametric FE model is built by APDL (ANSYS Parametric Design Language) and employed to perform sensitivity analysis. Effects of design variables $D, N, d_{1}, d_{2}$ on the objective function $f$ and constraint performance $\left(S_{t}, L_{t}\right)$ are obtained by sensitivity analysis.

2) Instead of obtaining $\left(S_{t}, L_{t}\right)$ by analytical or numerical techniques in the optimization loops, RSM is introduced to construct performance functions $g_{1}$ and $g_{2}$. This is a key step in proposed optimization approach and directly relates to the precision of final optimal design. Central composite designs (CCDs) method [11] is adopted to design 'numerical experiments', also known as sampling. Each sample is a combination of the four design variables $\left(D, N, d_{1}, d_{2}\right)$ with different values. Limited numbers of samples selected from the entire design space are computed by commercial FE software ANSYS and $\left(S_{t}, L_{t}\right)$ are extracted for each sample. All the samples $\left(D, N, d_{1}, d_{2}\right)$ with corresponding $\left(S_{t}, L_{t}\right)$ are then employed to build the full quadratic response surface model for $g_{1}$ and $g_{2}$. To evaluate the error between this approximation model and the FEA results, $\left(S_{t}{ }^{\prime}, L_{t}{ }^{\prime}\right)$ predicted by $g_{1}$ and $g_{2}$ are compared to $\left(S_{t}, L_{t}\right)$ calculated by ANSYS at each sample point and residuals are obtained.

3) MATLAB Optimization Toolbox ${ }^{\mathrm{TM}}$ is employed to implement the optimization procedure. Performance functions $g_{1}$ and $g_{2}$ are used as constraint functions. Both the gradient-based and Genetic Algorithm optimizers [17] are chosen to solve this formulated mathematical optimization problem and optimized results are compared. Further validations of these optimal results are done for constraint 
violations by FEA. Slight adjustments to the geometry may be needed to ensure these violations do not occur which can be based on the observations obtained from a sensitivity analysis to develop the final optimal design.

\section{Flexural properties of the composite truss}

\subsection{Experimental and numerical study}

The composite truss specimen used in three point bending test has a total length $(L)$ of $6 \mathrm{~m}$, triangular cross section circumscribing circle diameter $(D)$ of $348 \mathrm{~mm}$, bay number $(N)$ of 18 , longitudinal member diameter $\left(d_{1}\right)$ of $8.5 \mathrm{~mm}$ and the helical member diameter $\left(d_{2}\right)$ of $5.2 \mathrm{~mm}$. The total weight of the composite truss is $5.3 \mathrm{~kg}$. The longitudinal members have an average density $\left(\rho_{l}\right)$ of $2.092 \mathrm{~g} / \mathrm{cm}^{3}$ and axial tensile modulus $\left(E_{1}\right)$ of $45.8 \mathrm{GPa}$, while helical members have an average density $\left(\rho_{h}\right)$ of $2.099 \mathrm{~g} / \mathrm{cm}^{3}$ and axial tensile modulus $\left(E_{1}\right)$ of $46.6 \mathrm{GPa}$. The composite truss specimen is fabricated from glass fiber (GF) reinforced epoxy in the form of a prepreg tow: 2400 tex alkaline free glass fiber roving, Bisphenol-A epoxy resin CYD-128 (epoxy equivalent is 184 194, Epoxy Resin Division of Baling Petrochemical Co., Ltd.) [18] and curing agent diaminodiphenyl-methane (DDM) system. Just like in the composite IsoTruss ${ }^{\mathrm{TM}}$ [19] structures, a filament winding process has been employed to manufacture the triangular cross section composite truss specimen.

As shown in Fig. 3, a three-point bending test is performed on a universal testing machine (UTM). In the middle of composite truss specimen, the apex of triangular cross section is employed as the loading point. To measure the vertical displacement at the middle of composite truss, two electromechanical dial gauges 
are mounted at other vertices of the middle triangular cross section. The composite truss specimen is loaded at a rate of $1 \mathrm{~mm} / \mathrm{min}$. The data of the force sensor and electromechanical dial gauges are simultaneously recorded by the same data acquisition system.

To numerically simulate the three point bending test process, FEA is carried out using the general purpose finite element program ANSYS. A schematic view of the finite element model for the composite truss is shown in Fig. 4, which has the same geometry dimensions as the composite truss specimen used in three point bending test $\left(L=6 \mathrm{~m}, D=348 \mathrm{~mm}, N=18, d_{1}=8.5 \mathrm{~mm}, d_{2}=5.2 \mathrm{~mm}\right)$. A quadratic three node beam element BEAM189 is selected for the modeling. To simulate the simply supported boundary conditions, all the translational degrees of freedom at the four supporting nodes are fixed but they are rotationally free. The geometric non-linear option is switched on and an element size of $0.05 \mathrm{~m}$ is chosen for the finite element mesh.

Both the experimental and numerical results of the composite truss under threepoint bending test are plotted in Fig. 5. The two load-displacement curves are close to each other indicating that the flexural performance of the composite truss under three point bending can be simulated by FEA with adequate accuracy. Further examination of the graph shows that member buckling is initiated at $405 \mathrm{~N}$ which is the elastic limit $\left(S_{t}, L_{t}\right)$ of the load-displacement curve. Both the experimental and simulated curves consist of a turning point, elastic limit, and two linear parts, i.e. a bilinear curve. The slope $(k)$ of the first linear part defined by the elastic limit $k=L_{t} / S_{t}$ which is $33.75 \mathrm{~N} / \mathrm{mm}$, this is an indication of the structural stiffness of the composite truss. After the elastic limit, continued loading caused a rapid increase 
in the displacement but almost no change to the bearing load. The failure of the composite truss specimen occurs soon after the elastic limit is reached and is caused by the local buckling of the members. Consequently, the ultimate load is only slightly higher than the load which causes the structure to yield. As the elastic limit $\left(S_{t}, L_{t}\right)$ of the load-displacement curve indicates both the load bearing capacity and flexural stiffness of the lightweight composite truss, it can be considered as the most significant feature of this composite truss, under threepoint bending condition, and is chosen as structural response constraint for the optimal design.

\subsection{Sensitivity analysis}

To obtain the final optimal design of the composite truss, it is necessary to perform a sensitivity analysis of the design variables on the objective and constraint functions. In general, design sensitivity analysis is important to accurately determine the effects of changes to the design variables on the performance of composite structures. To evaluate these sensitivities efficiently and accurately, it is important to have appropriate techniques associated with good structural models [20]. A parametric finite element model is built in APDL in ANSYS and used to determine how the design variables $\left(D, N, d_{1}, d_{2}\right)$ affect the structural performance $\left(S_{t}, L_{t}\right)$. To ensure the loading point is located at mid span, the total bay number $(N)$ is constrained to be an even number. Eq. (8) is employed to evaluate effects of $(D$, $N, d_{1}, d_{2}$ ) on the objective function $f$. For the initial design, the following values are selected: $L=5.2 \mathrm{~m}, D=330 \mathrm{~mm}, N=18, d_{1}=8.5 \mathrm{~mm}, d_{2}=5.4 \mathrm{~mm}$. For practical considerations, $D$ varies from $190 \mathrm{~mm}$ to $650 \mathrm{~mm}$; $N$ is set as any even number between 10 and 44; and both $d_{1}$ and $d_{2}$ are from $4.0 \mathrm{~mm}$ to $10.0 \mathrm{~mm}$. Only one 
variable is changed at a time while the other three are maintained at the initial value. A load-displacement curve is obtained for each change and then the flexural performance of the composite truss structure is derived based on the effects of each individual design. The flow chart of sensitivity analysis by APDL is shown in Fig. 6. The effects of each design variable on the load-displacement curve of the composite truss under three-point bending are shown in Fig. 7. Corresponding to each curve and design variable, the elastic limit $\left(S_{t}, L_{t}\right)$ and structural weight $W$ are derived and plotted in Fig. 8 and Fig. 9 respectively.

As shown in Fig. 7 and Fig. 8, with an increase of $D, L_{t}$ increases while $S_{t}$ decreases which cause a distinct increase in structural stiffness $k$. It can be seen that the flexural properties of these lightweight composite truss structures are highly dependent on $D$ as the flexural stiffness and ultimate bearing capacity of the composite trusses are sensitive to changes in this value. Obvious increases of $S_{t}$ and $L_{t}$, with minimal changes of $k$, can be observed while $N$ varies from 10 to 44 . However, $N$ is considerably more effective in increasing the ultimate load $L_{t}$, even more so than $D$. This indicates that circumscribing circle diameter $D$ and bay number $N$ are critical to the integral flexural stiffness $k$ and ultimate load $L_{t}$ respectively. $S_{t}, L_{t}$ and $k$ will simultaneously increase with the increase of $d_{1}$ and $d_{2}$ from $4.0 \mathrm{~mm}$ to $10.0 \mathrm{~mm}$. However, $d_{1}$ is not as effective as $D$ and $N$ in increasing the values of $k$ and $L_{t}$. Compared with $d_{1}$, the increase of $d_{2}$ will also cause an increase of $L_{t}$ but with little effect on $k$. Structural weight $W$ is most sensitive to the change of $d_{2}$ although increasing any design variable will lead to the increase of $W$ (See Fig. 9).

\section{Response surface model of design constraints}


To save time and overcome convergence difficulties, which may be encountered due to highly distorted elements in large deformation nonlinear FE solutions, a response surface was employed for constraint evaluation in the optimization loops. The precision of the optimization solution depends only on the performance of the RSM which depends on the number of samples and the method used to select them.

Response surface models are multivariate polynomial models which can be written as:

$$
y(x)=a_{0}+\sum_{i=1}^{n} a_{i} x_{i}+\sum_{i<j}^{n} a_{i j} x_{i} x_{j}+\sum_{i=1}^{n} a_{i i} x_{i}^{2}+\cdots,
$$

where $a_{0}, a_{i}, a_{i j}$ and $a_{i i}$ are coefficients; $x_{i}$ and $x_{j}$ are variables. The model includes, from left to right, an intercept, linear terms, quadratic interaction terms, and squared terms. Higher order terms would follow, as necessary. Linear terms alone produce models with response surfaces that are hyper-planes. The addition of interaction terms allows for warping of the hyper-plane. Squared terms produce the simplest models in which the response surface has a maximum or minimum, and so an optimal response [11]. For this triangular cross section composite truss, a full quadratic response model is employed to construct performance functions $g_{1}$ and $g_{2}$ :

$$
\begin{aligned}
L_{t}= & g_{1}\left(D, N, d_{1}, d_{2}\right) \\
= & a_{0}+a_{1} D+a_{2} N+a_{3} d_{1}+a_{4} d_{2} \\
& +a_{5} D N+a_{6} D d_{1}+a_{7} D d_{2}+a_{8} N d_{1}+a_{9} N d_{2}+a_{10} d_{1} d_{2} \\
& +a_{11} D^{2}+a_{12} N^{2}+a_{13} d_{1}^{2}+a_{14} d_{2}^{2}
\end{aligned}
$$




$$
\begin{aligned}
S_{t}= & g_{2}\left(D, N, d_{1}, d_{2}\right) \\
= & b_{0}+b_{1} D+b_{2} N+b_{3} d_{1}+b_{4} d_{2} \\
& +b_{5} D N+b_{6} D d_{1}+b_{7} D d_{2}+b_{8} N d_{1}+b_{9} N d_{2}+b_{10} d_{1} d_{2} \\
& +b_{11} D^{2}+b_{12} N^{2}+b_{13} d_{1}^{2}+b_{14} d_{2}^{2}
\end{aligned}
$$

where $a_{i}, b_{i}(i=0,1,2, \ldots, 14)$ are coefficients to be determined by linear leastsquare fitting to sample data generated from numerical computation.

Although the precision of the response surface depends on the number of samples, a large quantity of samples will lead to significant computational costs.

Consequently, selecting an adequate amount of sample points which will produce acceptable accuracy in the regions of interest is vital for an accurate optimization procedure. To generate adequate sample points for fitting, central composite designs (CCDs), also known as Box-Wilson designs [11], are adopted to design numerical experiments. Table 1 shows sample sets of $D, N, d_{1}$ and $d_{2}$ patterned by 4 factors CCDs which allows 25 possible combinations of the 4 variables and 5 different values for each variable. For example, in the case of variable $D \in\left[D^{l}, D^{u}\right]$, $D^{l}, D^{u},\left(D^{u}+D^{l}\right) / 2,\left[D^{l}+\left(D^{u}+D^{l}\right) / 2\right] / 2$ and $\left[\left(D^{u}+D^{l}\right) / 2+D^{u}\right] / 2$ values of $-2,2,0,-1$ and 1 are selected for sampling in the given design space $\left[D^{l}, D^{u}\right]$. To increase the number of sample points further, resulting in improving the accuracy of response surface model, in addition to the given design space $(D \in[190,650], N \in[10,44]$, $\left.d_{1} \in[4.0,10.0], d_{2} \in[4.0,10.0]\right)$, an additional 3 sub regions $(D \in[200,400], N \in$ $[12,30], d_{1} \in[4.5,9.0], d_{2} \in[4.5,9.0] ; D \in[220,420], N \in[14,32], d_{1} \in[4.1,6.0], d_{2}$ $\left.\in[4.1,6.0] ; D \in[340,560], N \in[24,42], d_{1} \in[5.6,9.0], d_{2} \in[5.6,9.0]\right)$ are used for sampling. A total of 100 sample points are obtained and the parametric FE model used in the sensitivity analysis is employed to compute and extract the $\left(S_{t}, L_{t}\right)$ data for all the sample points. 
The 100 sample sets of $\left(D, N, d_{1}, d_{2}\right)$ and corresponding $\left(S_{t}, L_{t}\right)$ together with Eq. (10) and Eq. (11) lead to a linear least-square fitting problem which is implemented in the MATLAB Statistics Toolbox ${ }^{\mathrm{TM}}$ and the fitting coefficients of $g_{1}$ and $g_{2}$ are listed in Table 2. To assess the performance of the response surface model $g_{1}$ and $g_{2}$, the residual $(r)$ between the exact FE result $(y)$ and the results predicted by the response surface model $\left(y^{\prime}\right)$ is defined as:

$r=y-y^{\prime}$,

$r \%=\left(y-y^{\prime}\right) / y \times 100 \%$

Residuals between $\left(S_{t}, L_{t}\right)$ computed by FE simulation and $\left(S_{t^{\prime}}, L_{t}{ }^{\prime}\right)$ calculated by $g_{1}$, $g_{2}$ for all the 100 sample points are plotted in Fig. 10. Except for a few boundary condition sample points (sample points located at bounds of a given design space), most of the results predicted by $g_{1}, g_{2}$ have good accuracy with the precision of $g_{2}$ being higher than $g_{1}$.

\section{Optimization results}

After obtaining the performance function $g_{1}, g_{2}$ by RSM, the constrained nonlinear minimization solver 'fmincon' and the genetic algorithm solver 'ga' provided in MATLAB Optimization Toolbox ${ }^{\mathrm{TM}}$ are employed to solve this formulated optimization problem. 'fmincon' solver attempts to find a constrained minimum of a scalar function of several variables starting at an initial estimate. This is generally referred to as constrained nonlinear optimization. Genetic Algorithm solver 'ga' is used to solve both constrained and unconstrained optimization problems based on natural selection, the process that drives biological evolution. The algorithm begins by creating a random initial population and repeatedly modified a population of 
individual solutions. At each step, the algorithm selects individuals at random from current population to be parents and uses them to produce the children for the next generation. Over successive generation, the population "evolves" toward an optimal solution. The algorithm stops when stopping criteria is met.

Structural weight $W=f\left(D, N, d_{1}, d_{2}\right)$ expressed in Eq. (8) is specified as the nonlinear multivariable objective function to be minimized. As an example the minimum allowable ultimate load $L_{t}{ }^{p}$ and corresponding maximum allowable displacement $S_{t}^{p}$ are set as $1000 \mathrm{~N}$ and $20 \mathrm{~mm}$ respectively . Lower and upper bounds of $[190 \mathrm{~mm}, 650 \mathrm{~mm}],[10,44],[4.0 \mathrm{~mm}, 10.0 \mathrm{~mm}]$ and $[4.0 \mathrm{~mm}, 10.0 \mathrm{~mm}]$ given in constructing the response surface model of $g_{1}, g_{2}$ are imposed to $D, N, d_{1}$ and $d_{2}$ respectively. As prescribed by the MATLAB Optimization Toolbox ${ }^{\mathrm{TM}}$, the nonlinear inequality constraint $c$ and nonlinear equality constraint $c e q$ should be of the form $c \leq 0$ and $c e q=0$; the nonlinear constraint function in this case can be written as:

$c(1)=L_{t}^{p}-g_{1}\left(D, N, d_{1}, d_{2}\right) \leq 0$,

$c(2)=g_{2}\left(D, N, d_{1}, d_{2}\right)-S_{t}^{p} \leq 0$,

ceq $=N-2 \cdot$ round $(N / 2)=0$,

where Eq. (16) is the nonlinear equality constraint ensuring that $N$ is an even number.

One of the 100 sample points $\left(D, N, d_{1}, d_{2}\right)=(450 \mathrm{~mm}, 24,7.3 \mathrm{~mm}, 7.3 \mathrm{~mm})$ with satisfied constraints $\left(S_{t}, L_{t}\right)=(17.23 \mathrm{~mm}, 1098 \mathrm{~N})$ and minimum total structural weight of $W=9.57 \mathrm{~kg}$ is selected as the design for comparison. For the 'fmincon' 
solver, two algorithms 'active set' and 'interior point' are optional and both of them have been used to solve this optimization problem. Three types of termination criteria are provided: the maximum number of iterations, the tolerance of the design variables, and the tolerance of the function value. When one of these termination criteria is satisfied, the optimization iteration will be finished [17]. All the criteria are set as default. Different random combinations of $\left(D, N, d_{1}, d_{2}\right)$ in given bounds are used as starting points for the 'fmincon' optimization solutions. A starting point is not required by the 'ga' solver. Genetic algorithms allow global optimization but often lead to results that are only close to the global optimum. Final adjustments to the optimal results are required based on the observations obtained from the sensitivity analysis.

The optimal results obtained by both of the 'fmincon' and 'ga' solver with different options are listed in Table 3. The corresponding $\left(S_{t}, L_{t}\right)$ values checked for violations on all these optimal designs by FE simulation are also listed in the table. For all the 10 optimal results in Table 3, No. 8 has the minimum optimized structural weight $4.394 \mathrm{~kg}$ while satisfies performance constraints $\left(S_{t} \leq 20 \mathrm{~mm}, L_{t}\right.$ $\geq 1000 \mathrm{~N}$ ), thus it is selected as the temporary optimal design for the following adjustments. Helical member diameter $d_{2}$, which is the most sensitive to the structural weight, has already reached its lower bound and can not to be changed. As shown in Fig. 9, structural weight will decrease with decreases in $D, N$, and $d_{1}$. In order to further minimize weight, adjustments should be made to produce minimal $D, N$, and $d_{1}$ while satisfying the performance constraints. Compared with making changes in $d_{1}$, changes to $D$ and $N$ are more efficient in decreasing weight. Hence it is preferable to adjust $D$ and $N$ rather than $d_{1}$. For the temporary optimal design, $S_{t}$ 
$=19.78 \mathrm{~mm}$ is deemed to be close enough to the allowable value $20.00 \mathrm{~mm}$ while $L_{t}=1151.48 \mathrm{~N}$ is too far from the allowable value $1000 \mathrm{~N}$. Fig. 8 indicates that a decrease of $N$ will cause a decrease of $S_{t}$ and $L_{t}$ while decreasing $D$ will lead to a decrease in $L_{t}$ but an increase in $S_{t}$. Thus the first adjustment is to modify $N$ from 28 to 26 which led to a variation of $\left(S_{t}, L_{t}\right)$ from $(19.78 \mathrm{~mm}, 1151.48 \mathrm{~N})$ to $(17.63$ mm, $1033.89 \mathrm{~N})$. Further adjustments are made to $D$ from $391.91 \mathrm{~mm}$ to $378 \mathrm{~mm}$ resulting in the change of $\left(S_{t}, L_{t}\right)$ to $(18.19 \mathrm{~mm}, 1000.05 \mathrm{~N})$. As $L_{t}=1000.05 \mathrm{~N}$ is close enough to the allowable ultimate load $1000 \mathrm{~N}$, any further decrease on $D, N$, or $d_{1}$ will lead to a violation of the design constraints. Consequently, the design $(D$, $\left.N, d_{1}, d_{2}\right)=(378 \mathrm{~mm}, 26,8.61 \mathrm{~mm}, 4.00 \mathrm{~mm})$ with structural weight of $4.179 \mathrm{~kg}$ can be considered as the final optimal result. Compared to the initial design, a significant weight saving of $56 \%$ is achieved using this optimization approach.

\section{Conclusion}

A proposed multi-parameter optimization approach combining response surface methodology and a standard optimization program has been successfully applied to optimize the lightweight FRP composite truss structure under characteristic nonlinear structure response constraints. The composite triangular truss exhibited bilinear behavior under three-point bending test. The FEA result fits the experimental result well. A parametric FE model has been employed to perform sensitivity analysis and effects of the four design variables on the flexural performance of the composite truss are obtained. Response surface methodology has been used to construct performance functions of the composite truss structure under three-point bending and the result shows that flexural performance predicted by response surface model has good accuracy. Performance functions 
constructed by RSM are then employed by optimization program. Two different optimization solver 'fmincon' and 'ga' provided in MATLAB are used to solve this problem. Final optimal design is obtained after slight adjustments to design variables based on the results of sensitivity analysis. Significant savings in terms of weight (56\%) are achieved compared to the initial design. It indicates that coupling a response surface methodology with a standard optimization program leads to a useful tool suitable for the optimization of complex composite structure with nonlinear structure responses.

\section{References}

[1] António CC, Hoffbauer LN. An approach for reliability-based robust design optimisation of angle-ply composites. Compos Struct 2009;90:53-9.

[2] Schtüze R. Lightweight carbon fibre rods and truss structures. Mater Des 1997;18(4-6):231-8.

[3] Jensen DW, Weaver TJ. Mechanical characterization of a graphite/epoxy isotruss. J Aerosp Eng 2000;13(1):23-35.

[4] Jensen DW. A glimpse into the world of innovative composite IsoTruss structures. SAMPE J 2000;36(5):8-16.

[5] Rackliffe ME, Jensen DW, Lucas WK. Local and global buckling of ultralightweight IsoTruss structures. Compos Sci Technol 2006;66(2):283-8. 
[6] Paluch B, Grédiac M, Faye A. Combining a finite element programme and a genetic algorithm to optimize composite structures with variable thickness. Compos Struct 2008;83:284-94.

[7] Walker M, Smith RE. A technique for the multiobjective optimization of laminated composite structures using genetic algorithms and finite element analysis. Compos Struct 2003;62:123-8.

[8] Liu JS, Lu TJ. Multi-objective and multi-loading optimization of ultralightweight truss materials. Int J Solids Struct 2004;41:619-35.

[9] Abu-Odeh AY, Jones HL. Optimum design of composite plates using response surface method. Compos Struct 1998;43:233-42.

[10] Box GEP, Wilson KB. On the Experimental Attainment of Optimum Conditions (with discussion). J R Statist Soc B 1951;13(1):1-45.

[11] Khuri AI, Cornell JA. Response surfaces: designs and analyses. Marcel Dekker Inc.;1996.

[12] Chen NZ, Sun HH, Soares CG. Reliability analysis of a ship hull in composite material. Compos Struct 2003;62:59-66.

[13] Todoroki A, Ishikawa T. Design of experiments for stacking sequence optimizations with genetic algorithm using response surface approximation. Compos Struct 2004;64:349-57.

[14] Lanzi L, Giavotto V. Post-buckling optimization of composite stiffened panels: computations and experiments. Compos Struct 2006;73:208-20. 
[15] António CC. Optimisation of geometrically non-linear composite structures based on load-displacement control. Compos Struct 1999;46:345-56.

[16] Léné F, Duvaut G, Olivier-Mailhé M, Chaabane SB, Grihon S. An advanced methodology for optimum design of a composite stiffened cylinder. Compos Strut 2009;91:392-97.

[17] Park JS, Jung SN, Lee MK, Kim JM. Design optimization framework for tiltrotor composite wings considering whirl flutter stability. Compos Part B: Eng $2010 ; 41: 257-67$

[18] http://www.made-in-china.com/showroom/epoxyresin-baling/productdetailEobQaklHazcX/China-Epoxy-Resin-CYD-128-.html. 2010.09.07.

[19] McCune DT. Manufacturing quality of carbon/epoxy IsoTruss reinforced concrete structures. MS thesis. Brigham Young University; 2005.

[20] Youssif YG. Non-linear design and control optimization of composite laminated doubly curved shell. Compos Struct 2009;88:468-80. 


\section{Figure Captions}

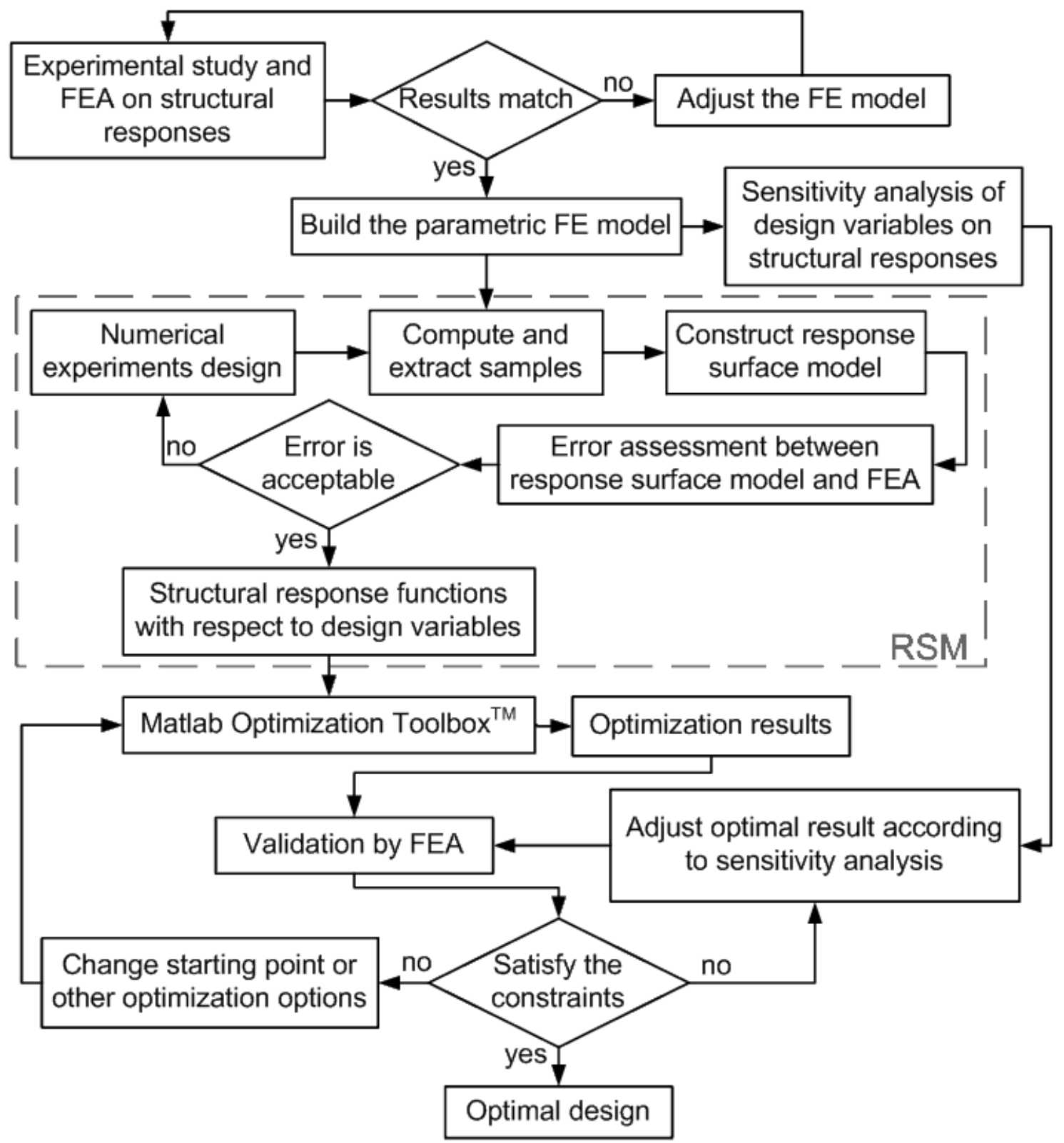

Fig. 1. Design optimization framework. 


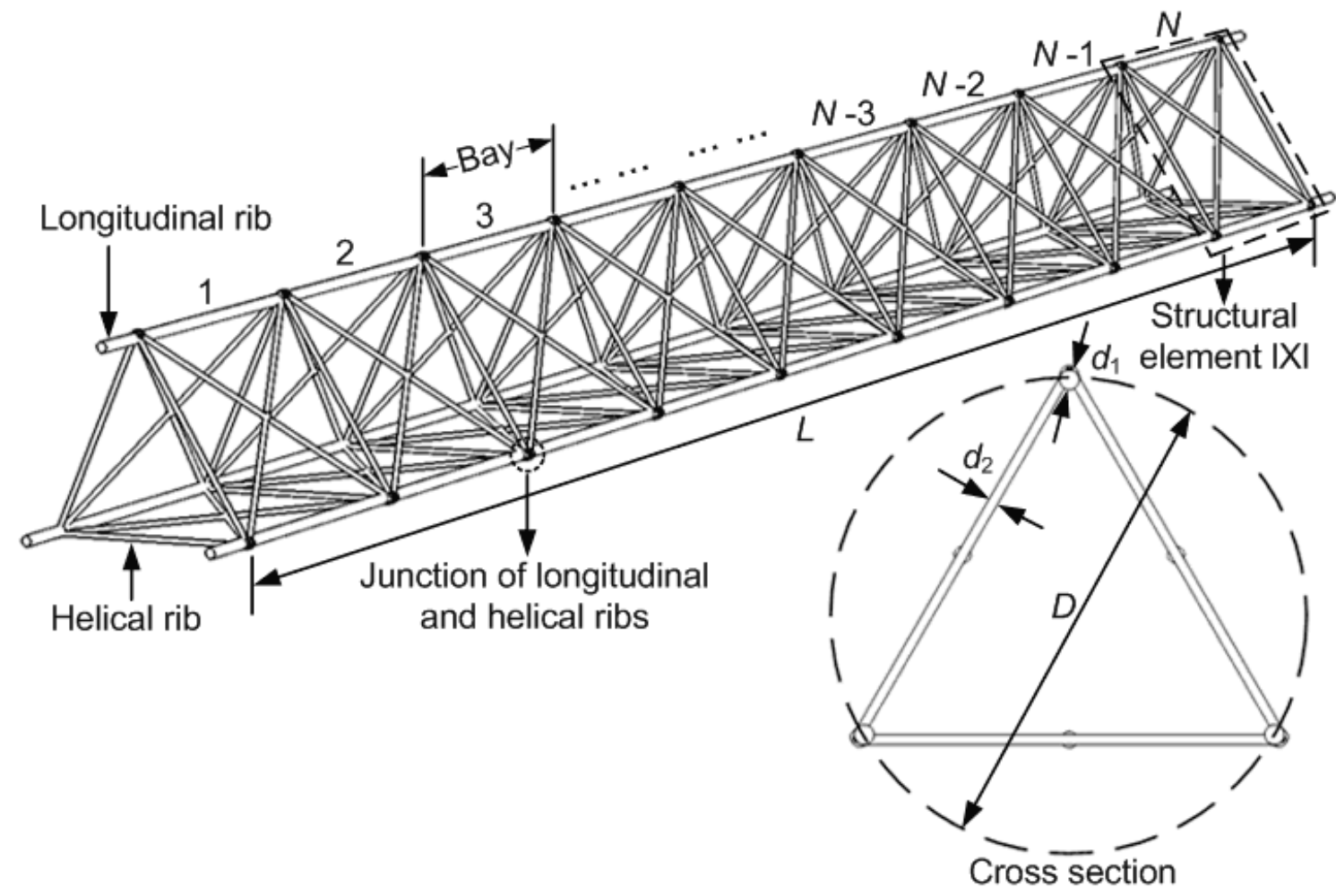

Fig. 2. Geometry of the triangular cross section lightweight FRP composite truss. 


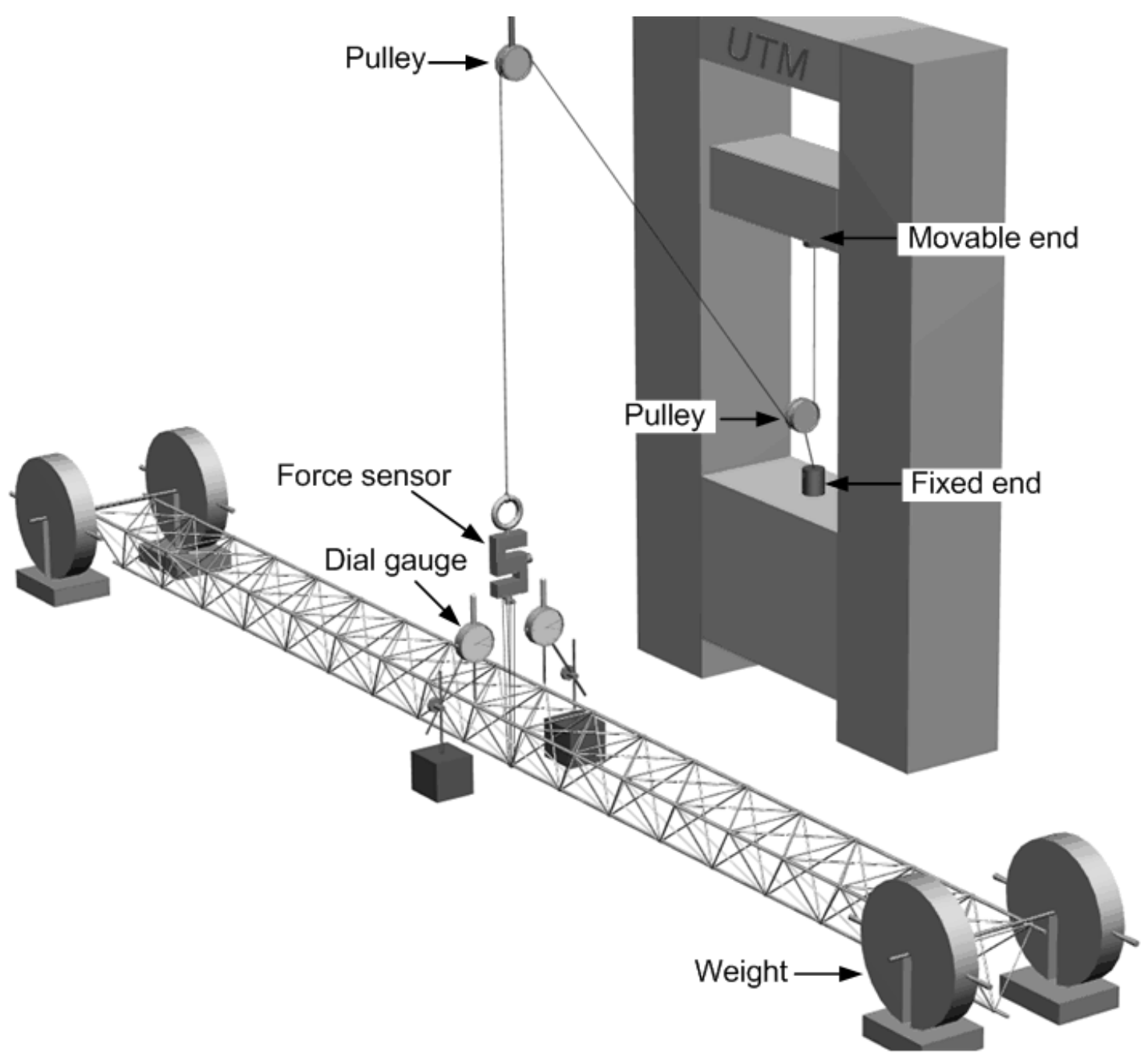

Fig. 3. Experimental setup for three-point bending test. 


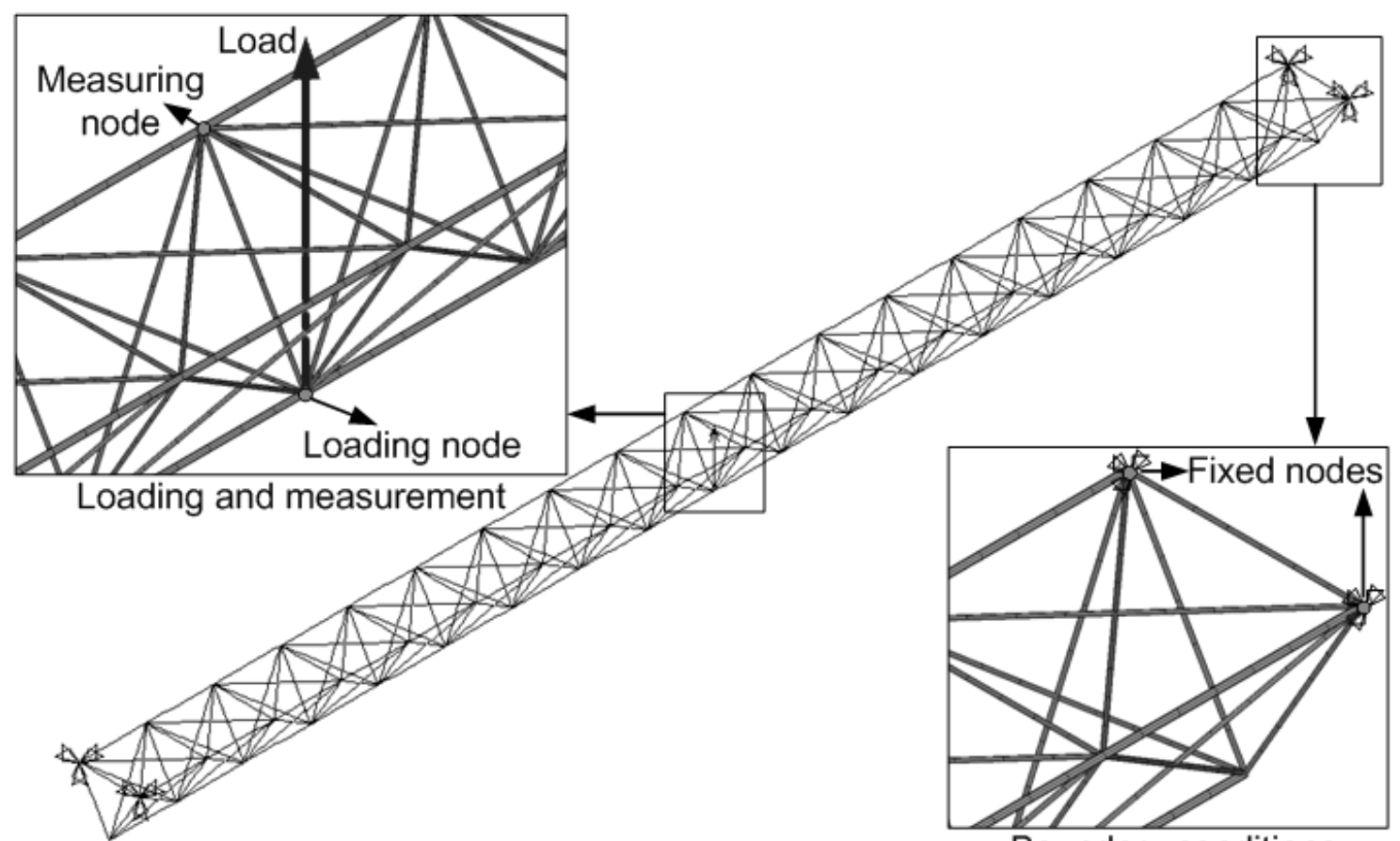

Boundary conditions

Fig. 4. FE model for the composite truss under three point bending. 


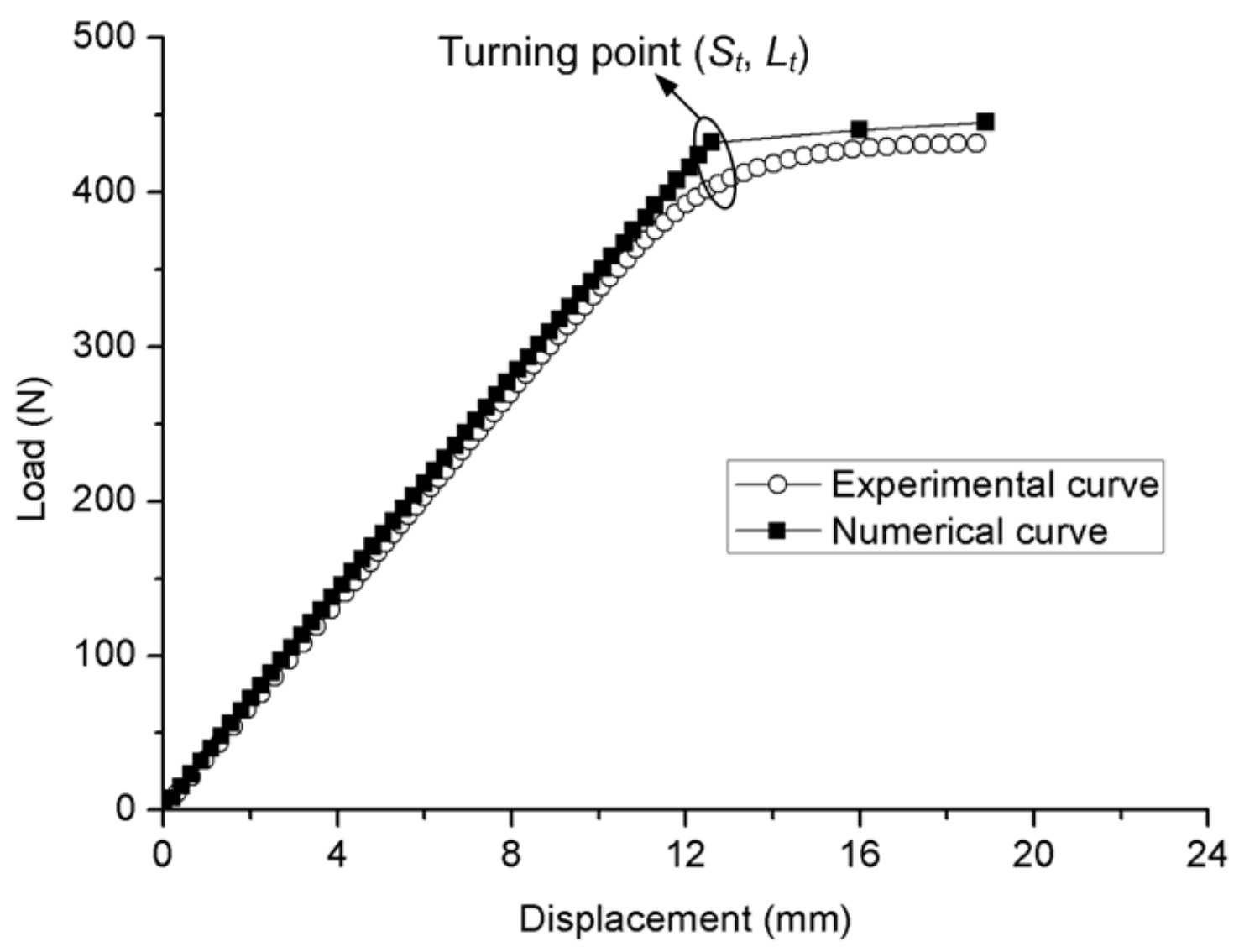

Fig. 5. Load versus displacement curve at the middle of composite truss under three-point bending. 


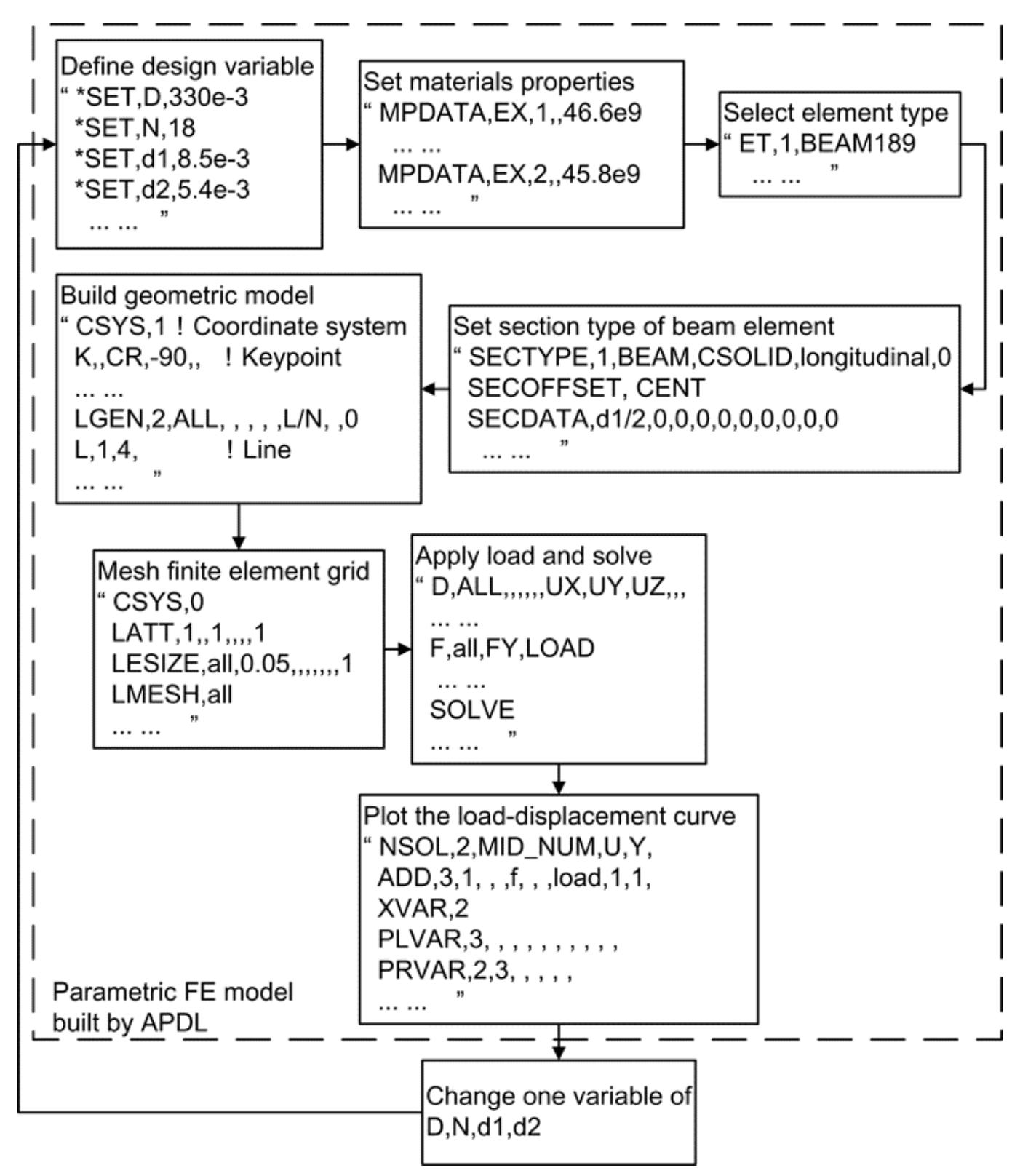

Fig. 6. Flow chart of the sensitivity analysis by APDL. 

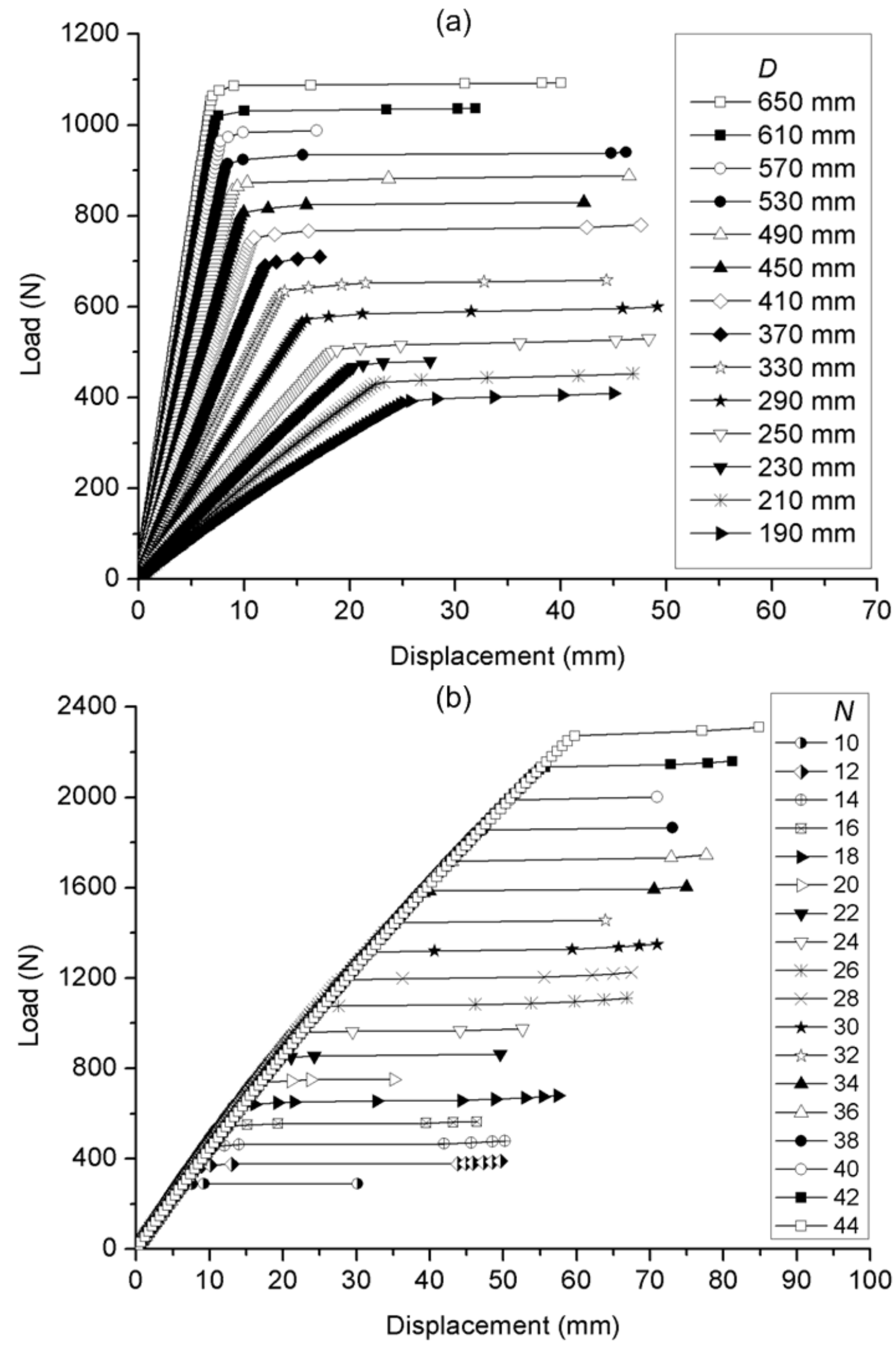

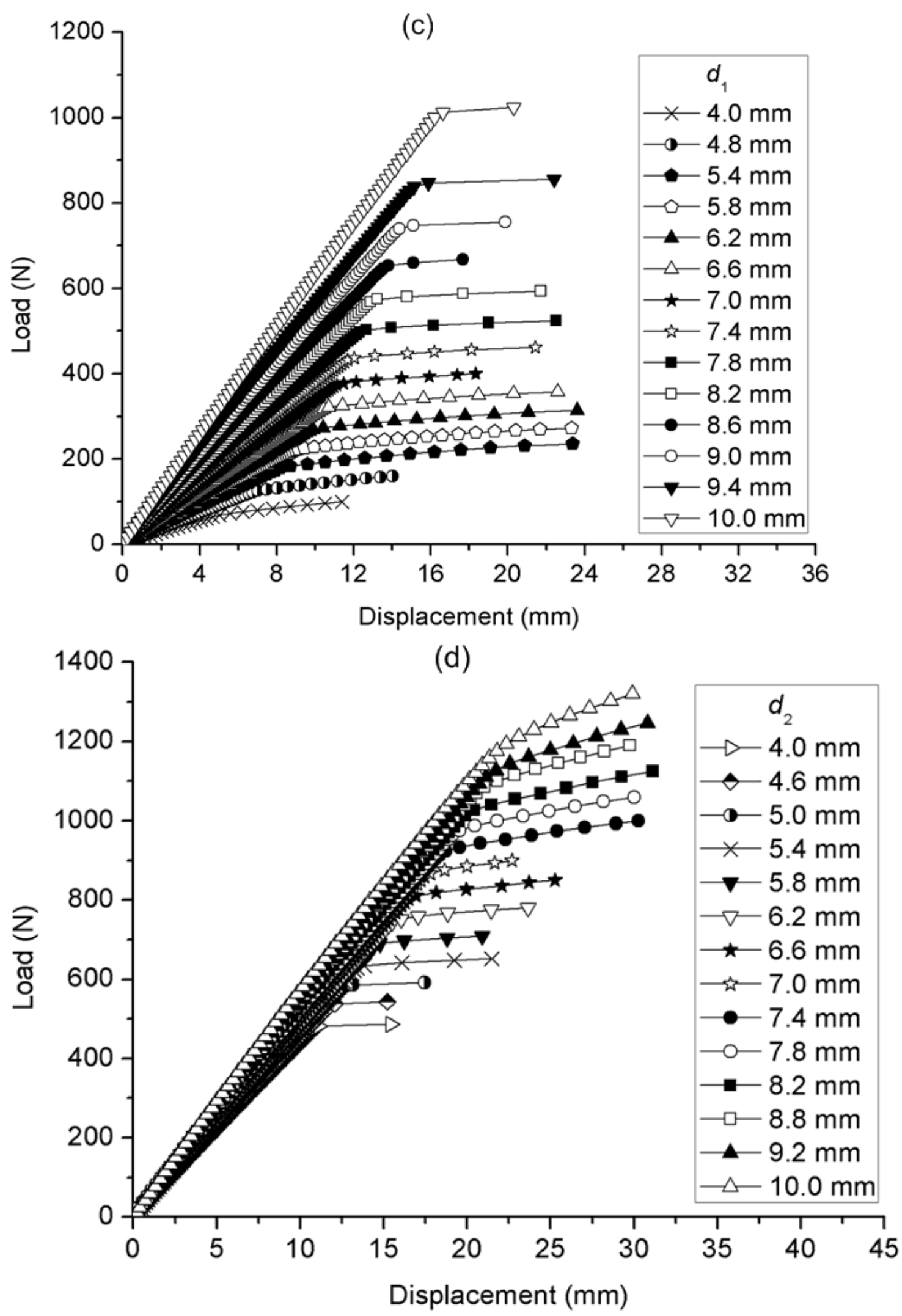

Fig. 7. Load-displacement curves of different design variables: (a) $D$; (b) $N$; (c) $d_{1}$ and (d) $d_{2}$. 

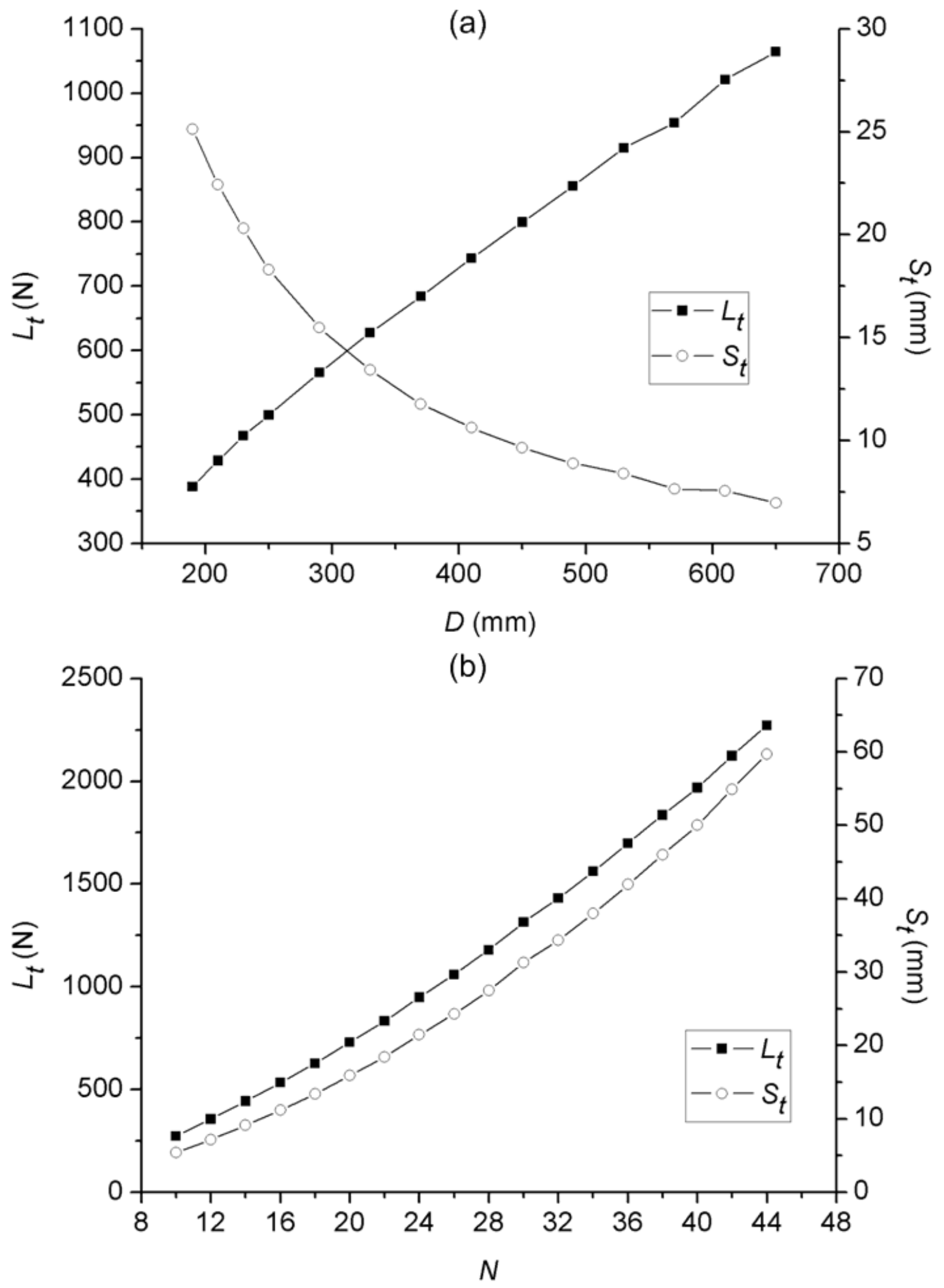

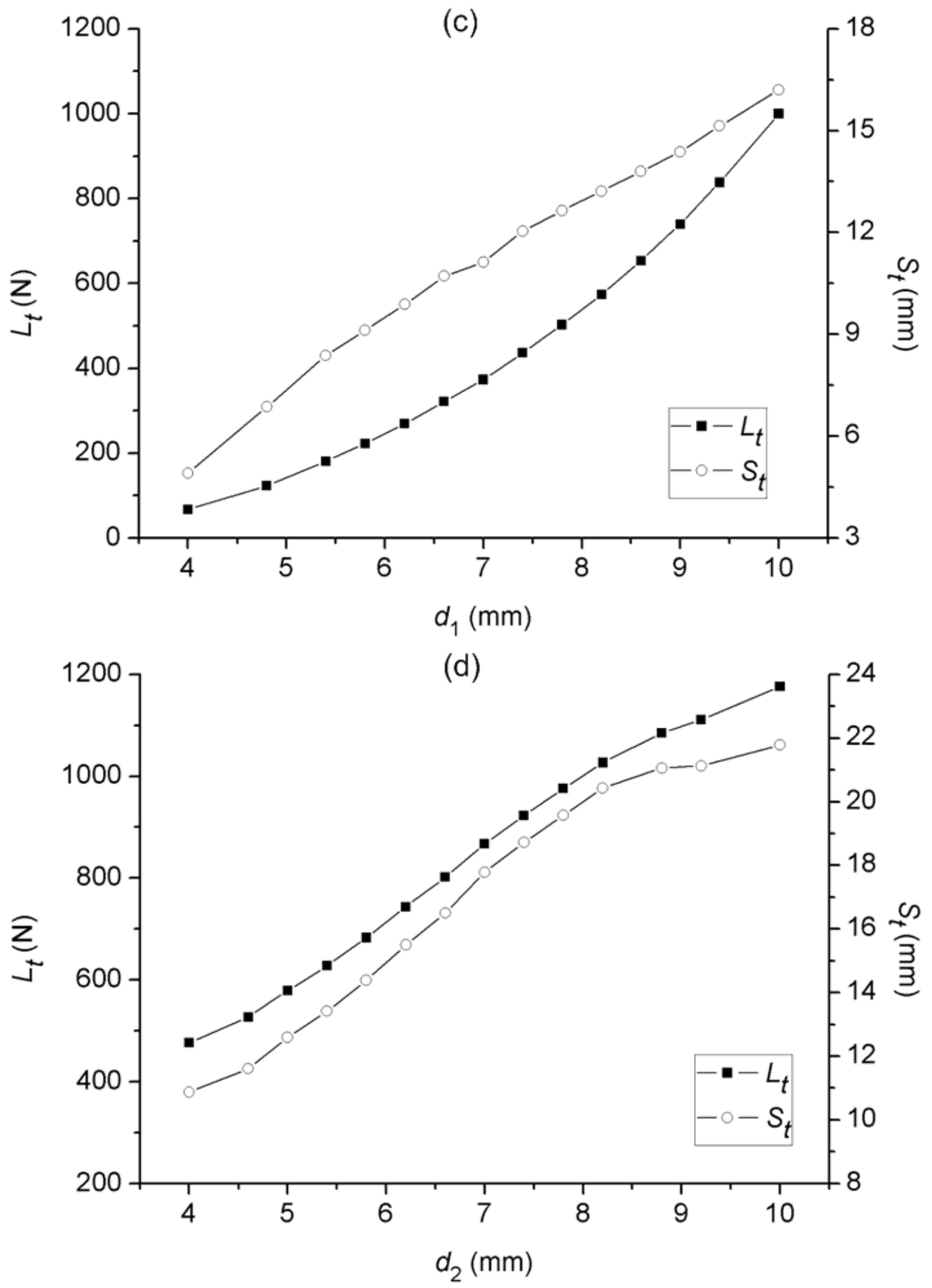

Fig. 8. Effects of individual design variable on $\left(S_{t}, L_{t}\right)$ : (a) $D$; (b) $N$; (c) $d_{1}$ and (d) $d_{2}$. 

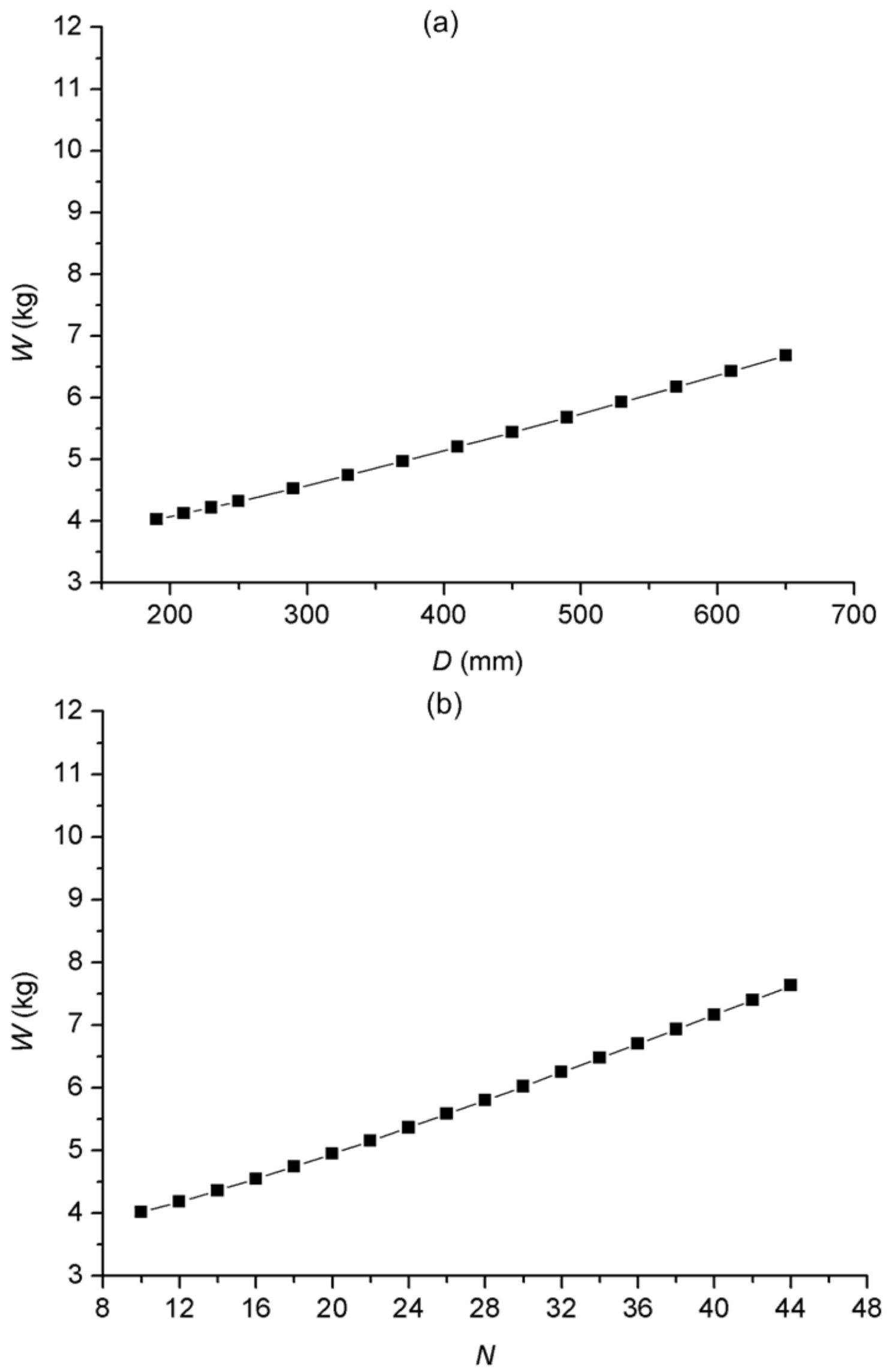

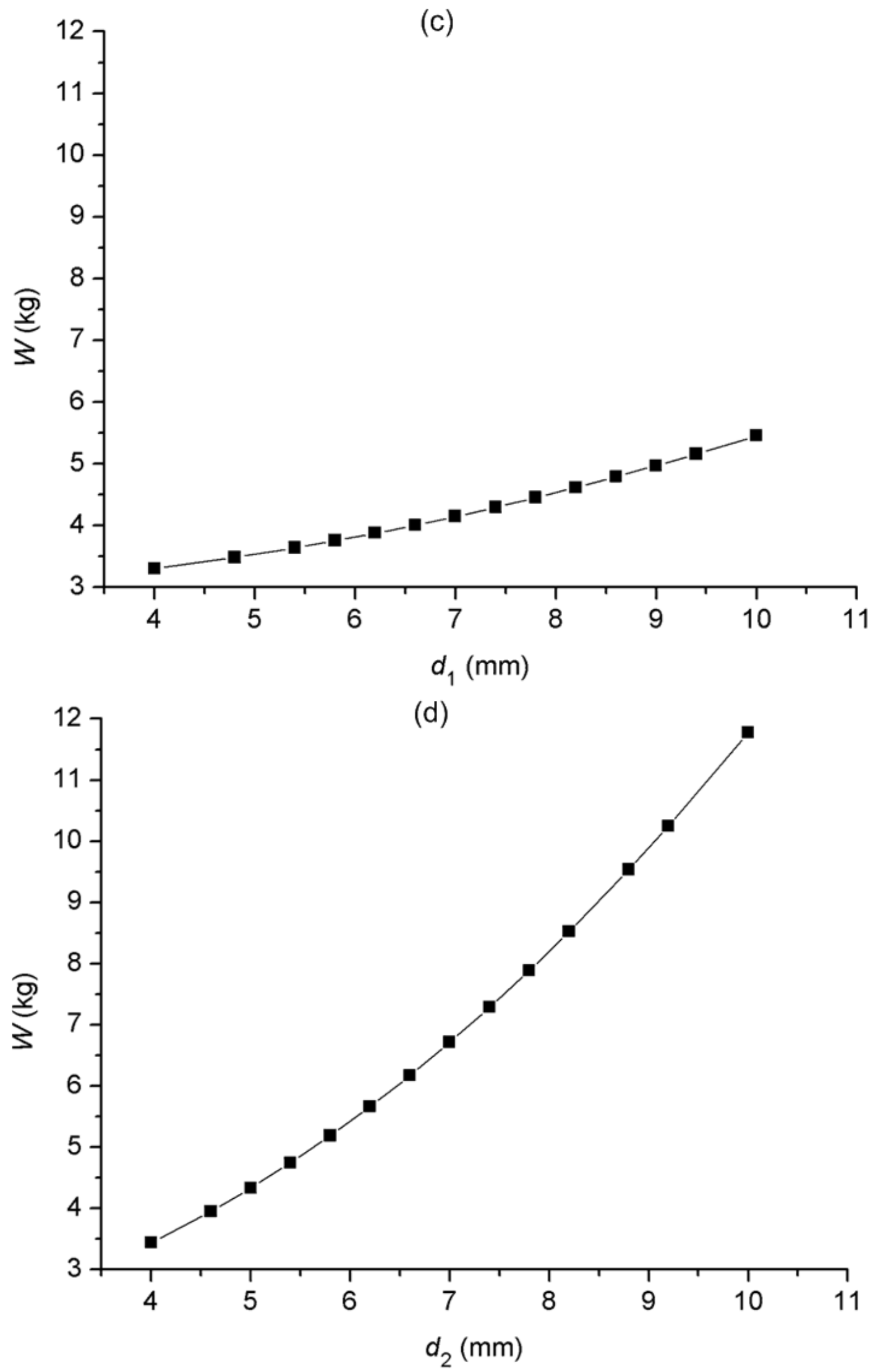

Fig. 9. Effects of individual design variable on $W$ : (a) $D$; (b) $N$; (c) $d_{1}$ and (d) $d_{2}$. 


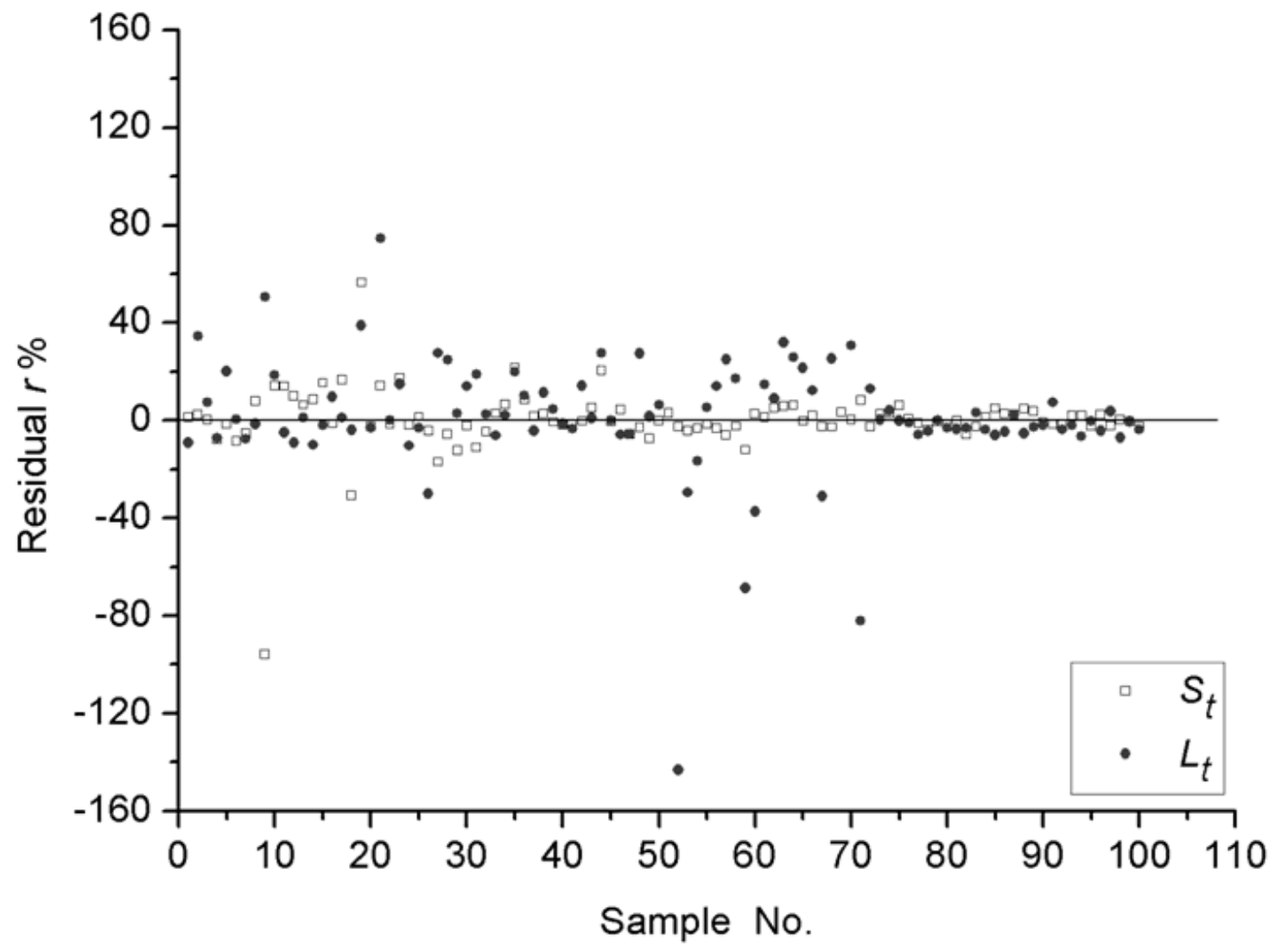

Fig. 10. Residuals for all the sample points.

Table 1

Sample sets of $\left(D, N, d_{1}, d_{2}\right)$ patterned by CCDs in given design space.

\begin{tabular}{|c|c|c|c|c|c|c|c|c|}
\hline \multirow{2}{*}{ No. } & \multicolumn{4}{|c|}{ CCDs } & \multirow{2}{*}{$\begin{array}{l}D(\mathrm{~mm}) \\
{[190,650]}\end{array}$} & \multirow{2}{*}{$\begin{array}{l}N \\
{[10,44]}\end{array}$} & \multirow{2}{*}{$\begin{array}{l}d_{1}(\mathrm{~mm}) \\
{[4.0,10.0]}\end{array}$} & \multirow{2}{*}{$\begin{array}{l}d_{2}(\mathrm{~mm}) \\
{[4.0,10.0]}\end{array}$} \\
\hline & $x_{1}$ & $x_{2}$ & $x_{3}$ & $x_{4}$ & & & & \\
\hline 1 & -1 & -1 & -1 & -1 & 305 & 20 & 5.5 & 5.5 \\
\hline 2 & -1 & -1 & -1 & 1 & 305 & 20 & 5.5 & 8.5 \\
\hline 3 & -1 & -1 & 1 & -1 & 305 & 20 & 8.5 & 5.5 \\
\hline 4 & -1 & -1 & 1 & 1 & 305 & 20 & 8.5 & 8.5 \\
\hline 5 & -1 & 1 & -1 & -1 & 305 & 36 & 5.5 & 5.5 \\
\hline 6 & -1 & 1 & -1 & 1 & 305 & 36 & 5.5 & 8.5 \\
\hline 7 & -1 & 1 & 1 & -1 & 305 & 36 & 8.5 & 5.5 \\
\hline 8 & -1 & 1 & 1 & 1 & 305 & 36 & 8.5 & 8.5 \\
\hline 9 & 1 & -1 & -1 & -1 & 535 & 20 & 5.5 & 5.5 \\
\hline 10 & 1 & -1 & -1 & 1 & 535 & 20 & 5.5 & 8.5 \\
\hline 11 & 1 & -1 & 1 & -1 & 535 & 20 & 8.5 & 5.5 \\
\hline 12 & 1 & -1 & 1 & 1 & 535 & 20 & 8.5 & 8.5 \\
\hline 13 & 1 & 1 & -1 & -1 & 535 & 36 & 5.5 & 5.5 \\
\hline
\end{tabular}




\begin{tabular}{rrrrrrlll}
14 & 1 & 1 & -1 & 1 & 535 & 36 & 5.5 & 8.5 \\
15 & 1 & 1 & 1 & -1 & 535 & 36 & 8.5 & 5.5 \\
16 & 1 & 1 & 1 & 1 & 535 & 36 & 8.5 & 8.5 \\
17 & -2 & 0 & 0 & 0 & 190 & 28 & 7.0 & 7.0 \\
18 & 2 & 0 & 0 & 0 & 650 & 28 & 7.0 & 7.0 \\
19 & 0 & -2 & 0 & 0 & 420 & 10 & 7.0 & 7.0 \\
20 & 0 & 2 & 0 & 0 & 420 & 44 & 7.0 & 7.0 \\
21 & 0 & 0 & -2 & 0 & 420 & 28 & 4.0 & 7.0 \\
22 & 0 & 0 & 2 & 0 & 420 & 28 & 10.0 & 7.0 \\
23 & 0 & 0 & 0 & -2 & 420 & 28 & 7.0 & 4.0 \\
24 & 0 & 0 & 0 & 2 & 420 & 28 & 7.0 & 10.0 \\
25 & 0 & 0 & 0 & 0 & 420 & 28 & 7.0 & 7.0 \\
\hline
\end{tabular}

\section{Table 2}

Coefficients for constraint functions $g_{1}$ and $g_{2}$.

\begin{tabular}{lrllr}
\hline & \multicolumn{1}{c}{$g_{1}$} & & \multicolumn{1}{c}{$g_{2}$} \\
\cline { 1 - 2 } \cline { 5 - 5 }$a_{0}$ & 8266.4437 & & $b_{0}$ & 0.0085520500 \\
$a_{1}$ & -7920.1524 & & $b_{1}$ & 0.0405911773 \\
$a_{2}$ & -186.3750 & & $b_{2}$ & -0.0005812750 \\
$a_{3}$ & -1410746.1978 & & $b_{3}$ & -0.4639449479 \\
$a_{4}$ & -457042.9579 & & $b_{4}$ & -4.0941267061 \\
$a_{5}$ & 120.6124 & & $b_{5}$ & -0.0051143869 \\
$a_{6}$ & 814575.1836 & & $b_{6}$ & -15.0981499093 \\
$a_{7}$ & 324749.2878 & & $b_{7}$ & -9.8416083341 \\
$a_{8}$ & 19087.4212 & & $b_{8}$ & 0.2147312638 \\
$a_{9}$ & 6447.8101 & & $b_{9}$ & 0.2449830932 \\
$a_{10}$ & 70321179.7090 & & $b_{10}$ & 1217.4360850941 \\
$a_{11}$ & -1280.2942 & & $b_{11}$ & 0.2425572699 \\
$a_{12}$ & 0.3586 & & $b_{12}$ & 0.0000176626 \\
$a_{13}$ & 38017146.0323 & & $b_{13}$ & -266.0652872443 \\
$a_{14}$ & -12463074.8300 & & $b_{14}$ & -301.8074909760 \\
\hline
\end{tabular}

\section{Table 3}

Optimal results with different optimization solvers and final optimal design obtained by adjustments.

\begin{tabular}{|c|c|c|c|c|c|c|c|c|c|c|c|c|}
\hline \multirow{2}{*}{ No. } & \multirow{2}{*}{ Solver } & \multicolumn{4}{|c|}{ Starting point } & \multicolumn{4}{|c|}{ Optimal results } & \multicolumn{2}{|c|}{ FEA } & \multirow{2}{*}{$\begin{array}{l}\text { Weight } \\
W(\mathrm{~kg})\end{array}$} \\
\hline & & $D(\mathrm{~mm})$ & $N$ & $d_{1}(\mathrm{~mm})$ & $d_{2}(\mathrm{~mm})$ & $D(\mathrm{~mm})$ & $N$ & $d_{1}(\mathrm{~mm})$ & $d_{2}(\mathrm{~mm})$ & $S_{t}(\mathrm{~mm})$ & $L_{t}(\mathrm{~N})$ & \\
\hline 1 & fmincon & 500.00 & 30 & 6.00 & 6.00 & 348.20 & 30 & 8.59 & 4.00 & 24.84 & 1144.00 & 4.281 \\
\hline 2 & fmincon & 450.00 & 28 & 5.00 & 4.00 & 324.97 & 28 & 9.16 & 4.00 & 26.41 & 1200.33 & 4.293 \\
\hline 3 & fmincon & 350.00 & 32 & 8.00 & 7.00 & 369.29 & 32 & 8.09 & 4.00 & 23.78 & 1096.33 & 4.331 \\
\hline 4 & fmincon & 310.00 & 26 & 9.30 & 6.50 & 371.18 & 26 & 9.17 & 4.00 & 20.13 & 1192.78 & 4.401 \\
\hline 5 & fmincon & 550.00 & 26 & 7.50 & 5.50 & 325.24 & 26 & 9.58 & 4.00 & 24.91 & 1239.33 & 4.378 \\
\hline 6 & fmincon & 280.00 & 34 & 5.00 & 8.00 & 388.49 & 34 & 7.67 & 4.00 & 22.99 & 1054.13 & 4.429 \\
\hline 7 & fmincon & 320.00 & 24 & 9.00 & 5.50 & 364.51 & 24 & 9.68 & 4.00 & 18.64 & 1180.00 & 4.480 \\
\hline
\end{tabular}




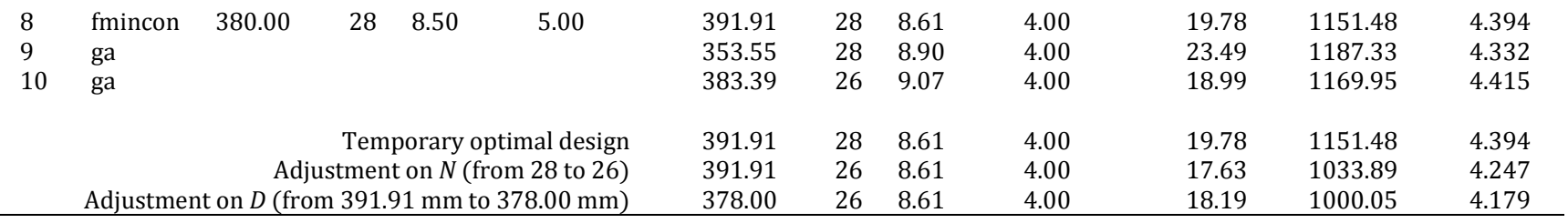

